\title{
Síndrome do pulmão encolhido: rara manifestação pulmonar do lúpus
}

\section{Shrinking lung syndrome: a rare lung manifestation of lupus}

Raimundo Noberto de Lima Neto ${ }^{1}$. Lindenberg Barbosa Aguiar².

1 Médico radiologista, São Carlos Imagem, Fortaleza, Ceará, Brasil. 2 Médico radiologista, Serviço de Radiologia e Diagnóstico por Imagem, Hospital Universitário Walter Cantídio (HUWC), Fortaleza, Ceará, Brasil.

\section{RESUMO}

Complicações torácicas em pacientes com lúpus eritematoso sistêmico são eventos comuns, notando-se acometimento dos mais diversos órgãos e compartimentos. No pulmão, podem-se observar pneumonias intersticiais, bronquiolite, vasculites, dentre outros, havendo grande contribuição dos exames de imagem para caracterização destas enfermidades, bem como para o diagnóstico diferencial com infecções. Uma complicação raramente descrita é a síndrome do pulmão encolhido, condição caracterizada por marcada redução volumétrica pulmonar, elevação das hemicúpulas diafragmáticas e padrão restritivo na espirometria. Esta doença ainda não tem a fisiopatologia totalmente descrita.

Relatamos um caso desta síndrome em paciente lúpica com dispneia progressiva, no qual foram empregados métodos de imagem tais como tomografia computadorizada do tórax e ultrassonografia das hemicúpulas, que demonstraram ausência de alterações parenquimatosas significativas e evidências de paralisia diafragmática à direita. Os achados foram adjuntos essenciais para a conclusão diagnóstica.

Palavras-chave: Lúpus eritematoso sistêmico. Paralisia diafragmática. Tomografia computadorizada. Ultrassonografia.

\section{ABSTRACT}

Thoracic complications in patients with systemic lupus erythematosus are common events, noticing involvement of many different organs and compartments. In the lung, it can be observed interstitial pneumonia, bronchiolitis and vasculitis, among others. There is great contribution of imaging studies to characterize these disorders as well as for the differential diagnosis with infections. A complication rarely described is the "shrinking lung" syndrome, a condition characterized by marked reduction in lung volume, elevated hemidiaphragms and restrictive pattern on spirometry.

We report a case of this syndrome in a lupus female patient with progressive dyspnea, in which were used imaging methods such as computed tomography of the chest and ultrasound of diaphragm, which demonstrated a normal parenchyma and evidence of diaphragmatic paralysis on the right. The findings were essential adjuncts to the diagnostic conclusion.

Keywords: Lupus erythematosus, systemic. Diaphragmatic paralysis. Computed tomography. Ultrasonography.

Autor correspondente: Raimundo Noberto de Lima Neto, Avenida Parnamirim, 1001, Mangabeira, Eusébio, Ceará. CEP: 61760-000. Telefone: +55 85 99673-3776. E-mail: nobertor@yahoo.com.br

Conflito de interesses: Não há qualquer conflito de interesses por parte de qualquer um dos autores.

Recebido em: 13 Fev 2018; Revisado em: 14 Mai 2018; Aceito em: 14 Mai 2018. 


\section{INTRODUÇÃO}

Em algum momento durante a história natural da doença, a maioria dos pacientes com lúpus eritematoso sistêmico (LES) apresentará algum sinal de envolvimento do parênquima pulmonar, sua vascularização, das superfícies pleurais e/ou do diafragma. ${ }^{1-3}$ Pleurisia, tosse e/ou dispneia são muitas vezes as primeiras pistas para envolvimento pulmonar ou mesmo para o próprio diagnóstico de lúpus em $\mathrm{si}^{4}{ }^{4} \mathrm{Em}$ alguns casos, no entanto, os testes de função pulmonar - como a capacidade de difusão do monóxido de carbono - e/ou exames de imagem anormais podem ser detectados em pacientes assintomáticos. ${ }^{5}$ Dentre os diagnósticos mais comuns, destacam-se a pleurite, as pneumonias intersticiais, bronquiolites, vasculites e a hipertensão pulmonar. Tromboembolsimo pulmonar normalmente ocorre quando se associa a síndrome do anticorpo antifosfolipídio. A síndrome do pulmão encolhido é uma rara manifestação pulmonar do LES. ${ }^{1}$

Os pacientes com LES e envolvimento pulmonar devem ser sempre avaliados para doenças infecciosas, especialmente aquelas causadas por bactérias ou vírus. Como muitos estão imunodeprimidos, tuberculose, infecções fúngicas e outras infecções oportunistas devem também ser consideradas no diagnóstico diferencial. ${ }^{6}$

Os exames de imagem têm papel fundamental na caracterização dos mais diversos tipos de acometimento pulmonar das doenças do colágeno, inclusive lúpus, bem como no seu diagnóstico diferencial com as supracitadas infecções. ${ }^{7}$ Particularmente no caso relatado, a demonstração ecográfica da paralisia diafragmática e a ausência de alterações parenquimatosas à tomografia foram essenciais para a elucidação diagnóstica.

\section{RELATO DO CASO}

Paciente de 37 anos, sexo feminino, com diagnóstico de lúpus eritematoso sistêmico desde 2007, complicado por nefrite lúpica em 2013, queixando-se de dispneia progressiva, atualmente apresentando-se aos mínimos esforços.

Internou-se no Hospital Universitário Walter Cantídio em setembro de 2014 para investigação diagnóstica. Na admissão, seu esquema terapêutico consistia de pulsos de ciclofosfamida, prednisona e cloroquina. Ao exame físico, apresentava-se discretamente dispneica. A ausculta cardíaca não mostrou alterações, porém, a pulmonar evidenciou redução do murmúrio vesicular em ambas as bases, sem ruídos adventícios. Ao ar ambiente, a saturação era de 96\%.

Os exames laboratoriais foram significativos apenas para anemia normocítica e normocrômica, atribuível à doença de base. O ecocardiograma transtorácico foi reportado como dentro dos limites da normalidade. Realizou-se espirometria que mostrou distúrbio ventilatório restritivo acentuado, com marcada redução da capacidade pulmonar total (Tabelas 1 e 2).

Tabela 1. Espirometria antes e após uso de bronodilatador.

\begin{tabular}{|c|c|c|c|c|c|c|c|c|}
\hline \multicolumn{9}{|c|}{ Espirometria } \\
\hline & & Pré-Bd & Ref & Limites inferior e superior & $\% \operatorname{Ref}$ & Pós-Bd & $\%$ Ref & $\%$ Alt \\
\hline $\mathrm{CVF}$ & $\mathrm{L}$ & 1,06 & 3,16 & $2,5-3,8$ & 34 & 1,07 & 34 & 0 \\
\hline VEF1 & $\mathrm{L}$ & 0,9 & 2,67 & $2,1-3,3$ & 34 & 0,88 & 33 & -2 \\
\hline VEF1/CVF & $\%$ & 85 & 84 & $75,9-92,9$ & & 83 & & \\
\hline
\end{tabular}

Legenda: CVF: capacidade vital forçada; VEF1: volume expiratório forçado no primeiro segundo; Pós-Bd: após o uso de broncodilatador; Pré-Bd: antes do uso de broncodilatador; Ref: valor de referência; Alt: percentual de alteração; L: litros.

Tabela 2. Volumes pulmonares antes e após uso de bronodilatador.

\begin{tabular}{|c|c|c|c|c|c|c|c|c|}
\hline \multicolumn{9}{|c|}{ Volumes pulmonares } \\
\hline & & Pré-Bd & Ref & Limites inferior e superior & $\% \operatorname{Ref}$ & Pós-Bd & $\% \operatorname{Ref}$ & $\%$ Alt \\
\hline $\mathrm{CPT}$ & $\mathrm{L}$ & 1,77 & 4,43 & $(3,4-5,5)$ & 40 & 1,88 & 42 & 6 \\
\hline VRE & $\mathrm{L}$ & 0,14 & & & & 0,09 & & -36 \\
\hline VR & $\mathrm{L}$ & 0,6 & 1,32 & $(0,5-2,1)$ & 46 & 0,61 & 46 & 1 \\
\hline $\mathrm{VR} / \mathrm{CPT}$ & $\%$ & 34 & 30 & $(18,7-40,7)$ & & 32 & & \\
\hline
\end{tabular}

Legenda: CPT: capacidade pulmonar total; VRE: volume de reserva expiratório; VR: volume residual. Pós-Bd: após o uso de broncodilatador; Pré-Bd: antes do uso de broncodilatador; Ref: valor de referência; Alt: percentual de alteração; L: litros. 
Realizou-se tomografia computadorizada do tórax, que mostrou redução volumétrica pulmonar bilateral, com formação de atelectasias compressivas e elevação das hemicúpulas diafragmáticas, sem sinais de enfermidade intersticial (Figuras 1 a 4). Notavam-se ainda focos de espessamento e calcificação pericárdicos, provavelmente relacionados a pericardite lúpica crônica (Figura 5), e derrame pleural à direita de volume insignificante.

Ao exame ecográfico das hemicúpulas diafragmáticas, observou-se acentuada redução da motilidade da hemicúpula diafragmática direita na inspiração livre, inspiração profunda e sniff test. Na hemicúpula diafragmática esquerda, notou-se amplitude menor que o normal apenas na inspiração profunda. Não houve movimentação paradoxal em ambas (Figura 6).

Figura 1. Escanograma da tomografia computadorizada mostrando acentuada redução volumétrica pulmonar bilateralmente, com elevação das hemicúpulas diafragmáticas.

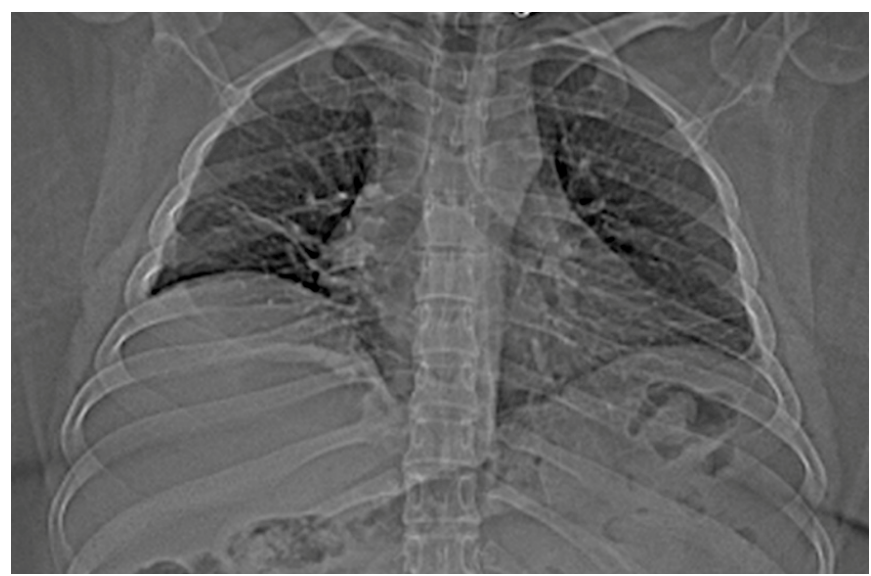

Figura 2. Reconstrução tomográfica no plano coronal, com janela para pulmão, mostrando acentuada redução volumétrica pulmonar (marcadamente dos lobos inferiores e médio), associada a elevação das hemicúpulas diafragmáticas.
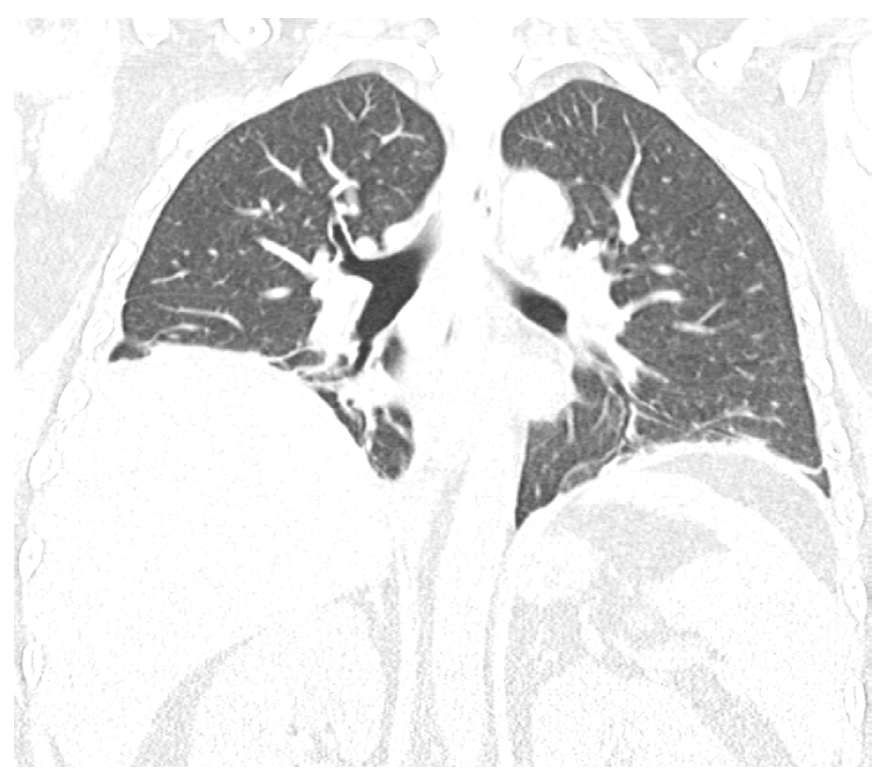

Figura 3. Reconstrução tomográfica no plano coronal, com janela para pulmão, mostrando atelectasias compressivas em ambos os lobos inferiores (setas).

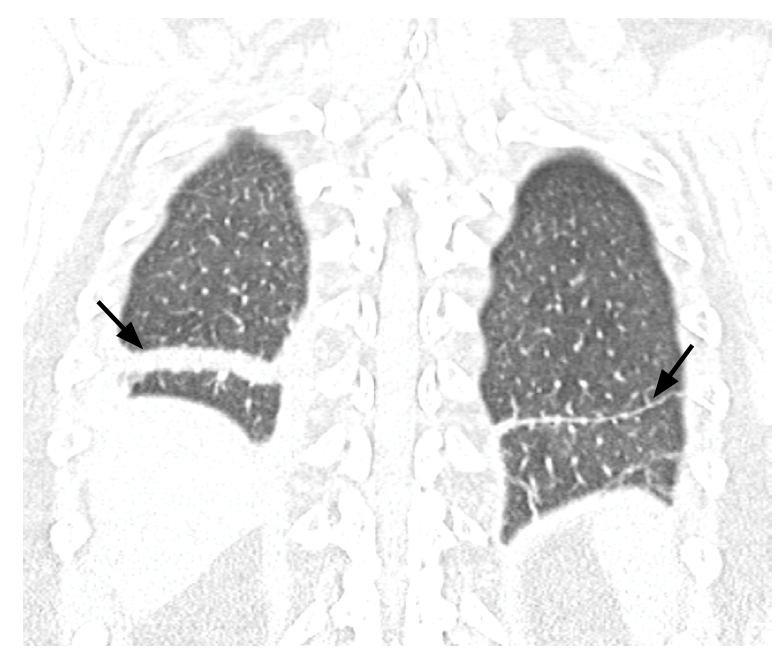

Figura 4. Tomografia computadorizada do tórax, com janela para pulmão, mostrando parênquima sem sinais de enfermidades intersticiais.

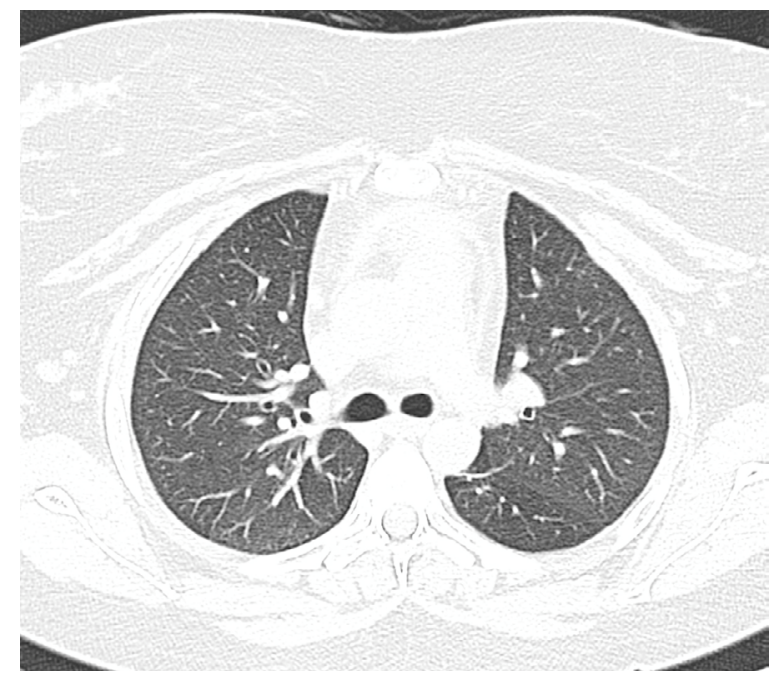

Figura 5. Reconstrução tomográfica no plano coronal, com janela para mediastino, mostrando pericárdio espessado e com focos de calcificação (setas).

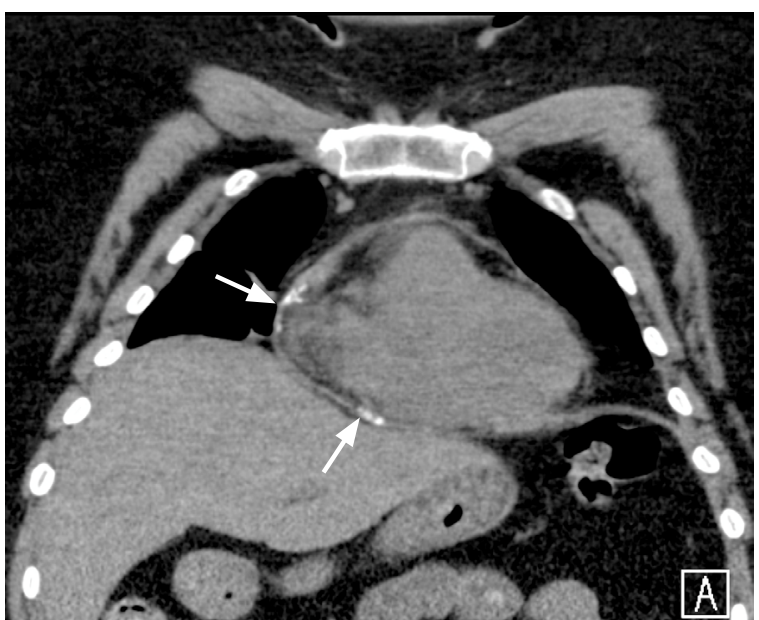


Figura 6. Exame ultrassonográfico das hemicúpulas diafragmáticas com emprego do modo M.

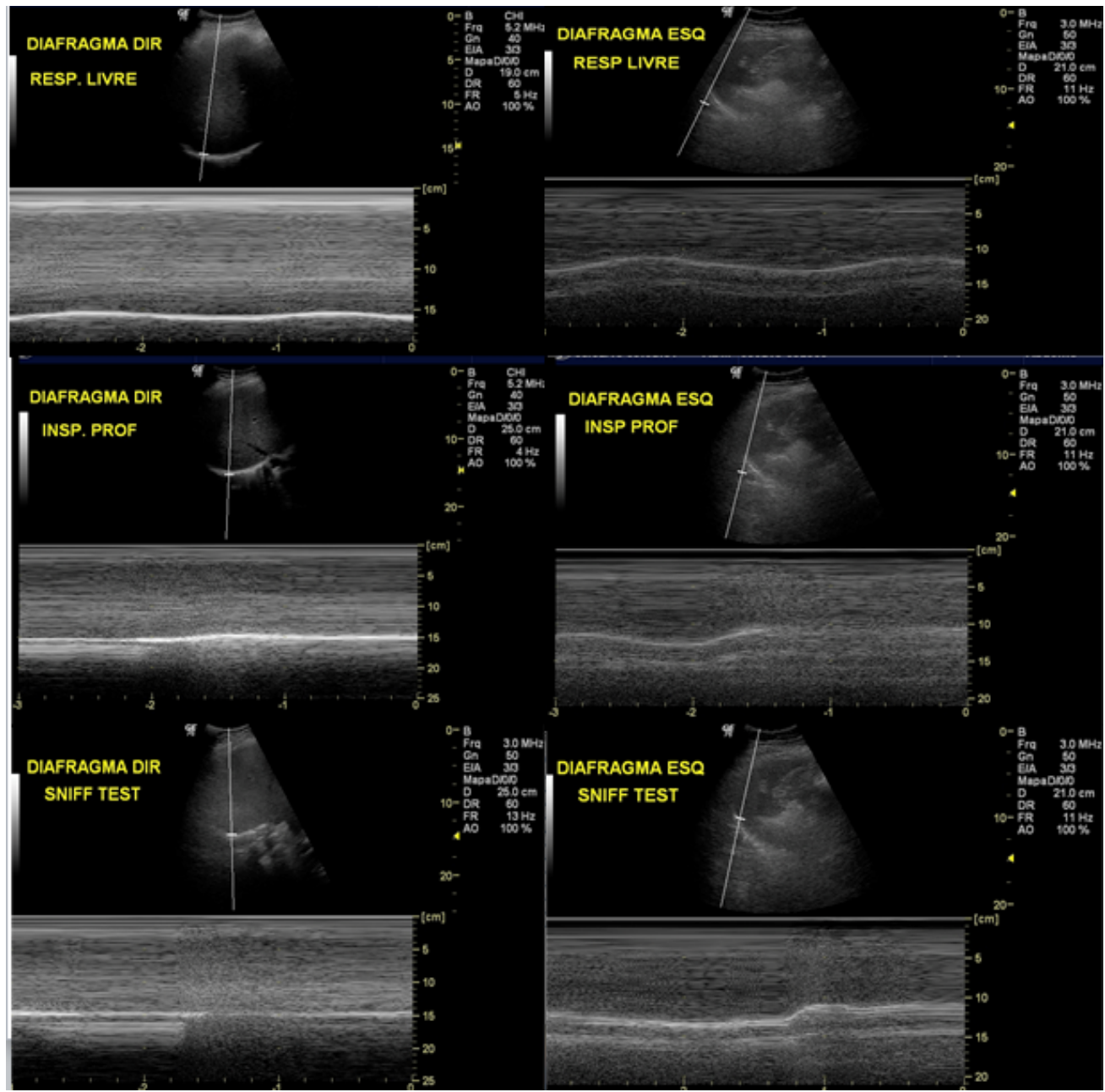

Nota: Na primeira coluna, nota-se motilidade reduzida da hemicúpula diafragmática direita. Na segunda coluna, nota-se motilidade normal da hemicúpula diafragmática esquerda, exceto na inspiração profunda, onde ainda se observa amplitude de movimentos reduzida.

\section{DISCUSSÃO}

A síndrome do pulmão encolhido (SPE), definida como uma perda progressiva do volume pulmonar associada a dispneia e pleurisia, foi primeiramente descrita em $1965 .{ }^{8}$ Esta síndrome é caracterizada por dispneia, episódios de dor pleurítica, diminuição progressiva nos volumes pulmonares, distúrbio ventilatório restritivo nos testes de função pulmonar e por ausência de evidências de fibrose intersticial ou de doença pleural significativa na tomografia computadorizada de tórax. Uma revisão da literatura identificou 77 pacientes com SPE e observou que $50(65 \%)$ tinham dor pleurítica. ${ }^{9}$

Resultados conflitantes foram encontrados em relação à patogênese subjacente a esta síndrome. Um possível mecanismo é uma miosite ou miopatia afetando o diafragma, resultando na elevação e mau funcionamento das hemicúpulas diafragmáticas. ${ }^{10,11}$ No entanto, outros relatos têm documentado a força muscular do diafragma como normal em pacientes com SPE e doença pulmonar restritiva. ${ }^{12}$ Outro modelo proposto sugere que a inflamação pleural crônica pode prejudicar a inspiração profunda e, posteriormente, levar à reorganização do parênquima, prejudicando a complacência pulmonar. ${ }^{13}$ Esta síndrome deve ser suspeitada em indivíduos com dispneia, radiografias de tórax normais e hemicúpulas diafragmáticas elevadas. $^{8}$ Deve-se fazer diagnóstico diferencial com outras causas de fraqueza ou paralisia diafragmática. Os glicocorticoides, teofilina e outras terapias imunossupressoras podem melhorar os sintomas e a função pulmonar. ${ }^{9,14}$

Os exames de radiografia convencional e de tomografia computadorizada do tórax normalmente mostram elevação das hemicúpulas diafragmáticas e atelectasias compressivas, sem evidências de enfermidades parenquimatosas que expliquem um distúrbio restritivo, como doenças intersticiais, por exemplo.

Como mencionado acima, observam-se casos em que a elevação das hemicúpulas diafragmáticas é decorrente de mau funcionamento deste músculo, com consequente paralisia. Basicamente todos os métodos de imagem possuem algum papel na avaliação da função diafragmática. Destacam-se aqueles que permitem avaliação dinâmica, como a fluoroscopia e o ultrassom. Este é preferido por sua 
portabilidade e pelo não uso de radiação ionizante, podendo ser utilizado em pacientes com restrição de movimentos, como aqueles internados em UTI. Trabalhos têm estabelecido os valores normais de amplitude de movimentação de ambas hemicúpulas diafragmáticas em inspiração livre, inspiração profunda e com sniff test (Tabela 3). ${ }^{15}$ Nas disfunções mais graves, pode-se observar, inclusive, movimentação paradoxal das hemicúpulas, com elevação durante a inspiração.

Tabela 3. Valores normais de amplitude de incursão das hemicúpulas diafragmáticas na inspiração.

\begin{tabular}{ccccc}
\hline & \multicolumn{2}{c}{$\begin{array}{c}\text { Hemicúpula } \\
\text { diafragmática direita }\end{array}$} & \multicolumn{2}{c}{$\begin{array}{c}\text { Hemicúpula } \\
\text { diafragmática esquerda }\end{array}$} \\
\hline Inspiração & Faixa, cm & Média, cm & Faixa, cm & Média, cm \\
\hline Livre & $0,26-2,1$ & 1,5 & $0,8-2,3$ & 1,58 \\
Profunda & $1,67-9,2$ & 5,69 & $3,8-9,6$ & 6,68 \\
Sniff test & $0,4-2,6$ & 1,66 & $0,76-2,7$ & 1,78 \\
\hline
\end{tabular}

Fonte: Gerscovich EO, Cronan M, McGahan JP, Jain K, Jones CD, McDonald C. Ultrasonographic evaluation of diaphragmatic motion. J Ultrasound Med. 2001 Jun;20(6):597-604.

Os exames de imagem têm papel fundamental no diagnóstico das complicações pulmonares do lúpus, desde a caracterização

\section{REFERÊNCIAS}

1. Orens JB, Martinez FJ, Lynch JP 3rd. Pleuropulmonary manifestations of systemic lupus erythematosus. Rheum Dis Clin North Am. 1994;20(1):159-93.

2. Keane MP, Lynch JP 3rd. Pleuropulmonary manifestations of systemic lupus erythematosus. Thorax. 2000;55(2):159-66.

3. Kim JS, Lee KS, Koh EM, Kim SY, Chung MP, Han J. Thoracic involvement of systemic lupus erythematosus: clinical, pathologic, and radiologic findings. J Comput Assist Tomogr. 2000;24(1):9.

4. Hellman DB, Kirsch CM, Whiting-O'Keefe Q, Simonson J, Schiller NB, Petri M, et al. Dyspnea in ambulatory patients with SLE: prevalence, severity, and correlation with incremental exercise testing. J Rheumatol. 1995;22(3):455.

5. Nakano M, Hasegawa H, Takada T, Ito S, Muramatsu Y, Satoh M, et al. Pulmonary diffusion capacity in patients with systemic lupus erythematosus. Respirology. 2002;7(1):45.

6. Rojas-Serrano J, Pedroza J, Regalado J, Robledo J, Reyes E, Sifuentes-Osornio J, et al. High prevalence of infections in patients with systemic lupus erythematosus and pulmonary haemorrhage. Lupus. 2008;17(4):295.

7. Capobianco J, Grimberg A, Thompson BM, Antunes VB, Jasinowodolinski D, Meirelles GS. Thoracic manifestations of collagen vascular diseases. Radiographics. 2012;32(1):33-50.

8. Hoffbrand BI, Beck ER. "Unexplained" dyspnoea and Shrinking de padrões específicos de doenças intersticiais, por exemplo, até o diagnóstico diferencial com infecções, que devem sempre ser lembradas, dada a imunodepressão sob a qual muitos se encontram. Paradoxalmente, numa suspeita de SPE, os exames radiológicos têm relevância quando não mostram alterações significativas do parênquima, excluindo outras pneumopatias que poderiam cursar com distúrbio restritivo. Especificamente na avaliação da motilidade das hemicúpulas diafragmáticas, os métodos de imagem como um todo têm bastante utilidade, com destaque para o ultrassom, por sua portabilidade e não uso de radiações ionizantes.

\section{CONCLUSÃO}

Não há critérios definidos para o diagnóstico da síndrome do pulmão encolhido. Entretanto, em qualquer paciente portador de lúpus eritematoso sistêmico com sintomas de dispneia progressiva, mostrando redução dos volumes pulmonares, elevação das hemicúpulas diafragmáticas, bem como evidências de padrão restritivo na espirometria, este diagnóstico deve ser considerado.

No caso relatado, lançou-se mão dos dados tomográficos e ecográficos como adjuvantes para o estabelecimento do diagnóstico de síndrome do pulmão encolhido numa paciente lúpica, uma complicação rara, porém, potencialmente reversível com o emprego de terapia imunossupressora.

lungs in systemic lupus erythematosus. Br Med J. 1965;1(5445):12737.

9. Toya SP, Tzelepis GE. Association of the Shrinking lung syndrome in systemic lupus erythematosus with pleurisy: a systematic review. Semin Arthritis Rheum. 2009;39(1):30-7.

10. Rubin LA, Urowitz MB. Shrinking lung syndrome in SLE: a clinical pathologic study. J Rheumatol. 1983;10(6):973-6.

11. Hardy K, Herry I, Attali V, Cadranel J, Similowski T. Bilateral phrenic paralysis in a patient with systemic lupus erythematosus. Chest. 2001;119(4):1274-7.

12. Laroche CM, Mulvey DA, Hawkins PN, Walport MJ, Strickland B, Moxham J, et al. Diaphragm strength in the Shrinking lung syndrome of systemic lupus erythematosus. Q J Med. 1989;71(265):429-39.

13. Hawkins P, Davison AG, Dasgupta B, Moxham J. Diaphragm strength in acute systemic lupus erythematosus in a patient with paradoxical abdominal motion and reduced lung volumes. Thorax. 2001;56(4):329-30.

14. Soubrier M, Dubost JJ, Piette JC, Urosevic Z, Rami S, Oualid T, et al. Shrinking lung syndrome in systemic lupus erythematosus. A report of three cases. Rev Rhum Engl Ed. 1995;62(5):395-8.

15. Gerscovich EO, Cronan M, McGahan JP, Jain K, Jones CD, McDonald C. Ultrasonographic evaluation of diaphragmatic motion. J Ultrasound Med. 2001;20(6):597-604.

\section{Como citar:}

Lima RN Neto, Aguiar LB. Síndrome do pulmão encolhido: rara manifestação pulmonar do lúpus. Rev Med UFC. 2018 out-dez;58(4):74-78. 